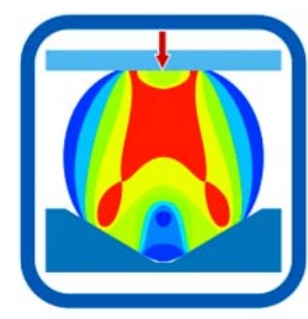

УДК 531.01+621.7.01

\section{РАЗДЕЛ I \\ МОДЕЛИРОВАНИЕ ПРОЦЕССОВ ОБРАБОТКИ ДАВЛЕНИЕМ}

DOI: $10.37142 / 2076-2151 / 2019-2(49) 3$

Алюшин Ю. А.

\title{
УСЛОВИЕ ПЛАСТИЧНОСТИ В ЭНЕРГЕТИЧЕСКОЙ МОДЕЛИ МЕХАНИКИ
}

Успешное использование обработки металлов давлением возможно только при научнообоснованном выборе параметров технологических процессов, обеспечивающих их устойчивость и эффективность. Для этого необходимо знать, какие факторы являются определяющими, как они влияют на изменение усилий формоизменения, характер течения металла, его свойства после деформации. На протяжении всей истории развития теории обработки металлов давлением выбор и аргументация условий перехода в пластическое состояние и предельных степеней деформации являются наиболее важными для обеспечения необходимого качества получаемых изделий.

Подробный исторический обзор условий пластичности от максимальных сдвиговых деформаций и нормальных напряжений до современных энергетических критериев приведен в работах [1-2]. В работах Зибеля Э., Закса Г. доказано, что для наступления пластического состояния и получения остаточных деформаций в материале в соответствии с его физическими свойствами должны быть преодолены сопротивления сдвигу, формоизменение материала в основном происходит за счет перемещения его частиц в плоскостях наибольших скалывающих напряжений. Работы Генки Г., Прандтля Л. послужили развитию теории напряженного и деформированного состояний.

В первой половине 20 века пониманию основных положений теории способствовала геометрическая интерпретация видов и условий напряженного состояния (круги Мора, поверхности пластичности). Распространению и развитию теории обработки давлением посвящены работы Попова Е. А., Томленова А. Д. и других российских ученых [2-5]. К концу 70-х годов окончательно сформировались инвариантные гипотезы максимальных интенсивностей напряжений и деформаций [4-6], которые получили экспериментальное подтверждение при различных схемах нагружения. В настоящее время общепринятым является критерий пластичности в виде условия постоянства удельной энергии, расходуемой на изменение формы частиц деформируемого материала, который имеет несколько других интерпретаций [6-7].

Цель работы - обосновать механизм перехода от обратимых деформаций к необратимым с использованием предельных значений интенсивности касательных напряжений и основных положений энергетической модели механики.

В настоящее время наибольшее распространение получили классические методы механики деформируемого твердого тела в пространстве переменных Эйлера, хотя, как отмечено в работе [7], «...с физической точки зрения переменные Лагранжа кажутся особенно удобными для описания движения сплошной среды».

Рассмотрим оба варианта с указанием их преимуществ и недостатков. В самом общем случае траектории перемещения частиц можно описать уравнениями:

$$
x=x(\alpha, \beta, \gamma, t), \quad y=y(\alpha, \beta, \gamma, t), \quad z=z(\alpha, \beta, \gamma, t),
$$


где $\alpha, \beta, \gamma$ - переменные Лагранжа. Объективные закономерности, которые являются предметом исследований, могут быть получены только на основе объективных аргументов. Так как выбор системы координат субъективен, основную информацию о состоянии частиц несут производные от координат по переменным Лагранжа, которые определяют тензоры деформации и скорости деформации в пространстве переменных Лагранжа:

$$
\begin{gathered}
\partial x_{i} / \partial \alpha_{p} \equiv x_{i, \alpha_{p}}=\left(\begin{array}{lll}
x_{\alpha} & x_{\beta} & x_{\gamma} \\
y_{\alpha} & y_{\beta} & y_{\gamma} \\
z_{\alpha} & z_{\beta} & z_{\gamma}
\end{array}\right), \\
\partial x_{i, t} / \partial \alpha_{p} \equiv x_{i, t \alpha_{p}}=\left(\begin{array}{ccc}
x_{t \alpha} & x_{t \beta} & x_{t \gamma} \\
y_{t \alpha} & y_{t \beta} & y_{t \gamma} \\
z_{t \alpha} & z_{t \beta} & z_{t \gamma}
\end{array}\right) .
\end{gathered}
$$

Энергетическая модель механики [8-9] ориентирована на использование уравнений (1) и основана на предположении, что любые закономерности должны быть следствием закона сохранения энергии, а энергия, как обобщенная скалярная функция любых видов движения, может быть представлена в виде линейной комбинации 13 независимых инвариантов $\xi_{i}$ уравнений движения в форме Лагранжа (1):

$$
\delta E=\sum_{i=1}^{13} \delta E_{i}\left(\xi_{i}\right)=\sum_{i=1}^{13} k_{i} \xi_{i} \delta V_{0},
$$

в том числе модули векторов перемещения, скорости и ускорения:

$$
\begin{aligned}
& \xi_{1}=|u|=\sqrt{u_{x}^{2}+u_{y}^{2}+u_{z}^{2}}=\sqrt{(x-\alpha)^{2}+(\mathrm{y}-\beta)^{2}+(\mathrm{z}-\gamma)^{2}}, \\
& \xi_{2}=|v|=\sqrt{v_{x}^{2}+v_{y}^{2}+v_{z}^{2}}=\sqrt{x_{t}^{2}+y_{t}^{2}+z_{t}^{2}}, \\
& \xi_{3}=|w|=\sqrt{w_{x}^{2}+w_{y}^{2}+w_{z}^{2}}=\sqrt{x_{t t}^{2}+y_{t t}^{2}+z_{t t}^{2}}, \quad \xi_{4}=s=\int_{t_{1}}^{t_{2}}|v| d t,
\end{aligned}
$$

три инварианта тензора деформации (2):

$$
\begin{gathered}
\xi_{5}=x_{\alpha}+y_{\beta}+z_{\gamma}, \\
\xi_{6}=x_{\alpha}^{2}+x_{\beta}^{2}+x_{\gamma}^{2}+y_{\alpha}^{2}+y_{\beta}^{2}+y_{\gamma}^{2}+z_{\alpha}^{2}+z_{\beta}^{2}+z_{\gamma}^{2}, \quad \xi_{7}=\left|x_{i, p}\right|=R=\delta V / \delta V_{0},
\end{gathered}
$$

три инварианта тензора скорости деформации (3):

$$
\xi_{8}=x_{t \alpha}+y_{t \beta}+z_{t \gamma}, \quad \xi_{9}=x_{t \alpha}^{2}+x_{t \beta}^{2}+x_{t \gamma}^{2}+y_{t \alpha}^{2}+y_{t \beta}^{2}+y_{t \gamma}^{2}+z_{t \alpha}^{2}+z_{t \beta}^{2}+z_{t \gamma}^{2}, \quad \xi_{10}=\left|x_{i, t \alpha_{p}}\right|,
$$

интегральные по времени инварианты путь $s$ и три интеграла по времени от трех инвариантов (7) тензора скорости деформации

$$
s=\int_{t_{1}}^{t_{2}} v \mathrm{~d} t, \quad \xi_{11}=\int \xi_{8} \mathrm{~d} t, \quad \xi_{12}=\int \xi_{9}^{1 / 2} \mathrm{~d} t, \quad \xi_{13}=\int \xi_{10}^{1 / 3} \mathrm{~d} t
$$

Поведение системы при самых разнообразных воздействиях определяет закон сохранения энергии, который для замкнутой системы принимает вид: 


$$
d \delta E=\delta V_{0}\left(d \sum_{i=1}^{13} k_{i} \xi_{i}\right)=0
$$

где $\xi_{i}$ - инварианты (2) - (8), $k_{i}$ - коэффициенты, обеспечивающие равенство размерности слагаемых в правой части уравнения (9) и характеризующие физические свойства материалов [8-9].

Так как для большинства задач необходимо учитывать взаимодействие рассматриваемой системы, в том числе бесконечно малой частицы, с окружающей средой, уравнение (9) следует дополнить двумя независимыми слагаемыми $\omega_{\mathrm{e}}$ и $\omega_{T}$, которые учитывают внешние механические (силовые) и температурные воздействия:

$$
d \delta E=\delta V_{0}\left(k_{1} \xi_{1, t}+k_{2} \xi_{2, t}+k_{4} \xi_{4, t}+k_{6} \xi_{6, t}+k_{9} \xi_{9, t}+k_{10} \xi_{10, t}+k_{12} \xi_{12, t}+k_{13} \xi_{13, t}-\omega_{e}-\omega_{T}\right) d t=0,
$$

где $\omega_{T}=c_{0} \rho_{0} T_{t}, c_{0}, \rho_{0}, T$ - теплоемкость, плотность и температура, соответственно. Слагаемое $\omega_{T}$ должно учитывать тепло, выделяемое из системы за счет диссипации при переходе от обратимых деформаций к необратимым.

Уравнение (10) эквивалентно первому началу термодинамики для частицы сплошной среды [10], в соответствии с которым работа внешних сил затрачивается на изменение кинетической, упругой или других видов энергии частицы. Все известные формы законов движения [11] следует рассматривать как частные случаи уравнения (10).

Энергетическая модель, используя пространство Лагранжа, значительно проще в математическом плане по сравнению с классическим вариантом, позволяет решать различные задачи в области обратимых и необратимых деформаций, выявлять особенности механизмов деформации с использованием внутренних источников энергии, например, при свободных и вынужденных колебаниях [12-13].

Вместе с тем ориентация на переменные Лагранжа, хотя это и более информативно [8-9], отталкивает большую часть исследователей, так как во всех университетах, как и в подавляющем большинстве учебников, преобладает классическая механика с описанием процессов движения и деформации в пространстве Эйлера.

В пространстве переменных Лагранжа, используемом в уравнениях (1), все рассматриваемые функции $f(t)$ относятся к фиксированными частицам, координаты которых являются аргументами этих функций. Производные от уравнений (1) по времени $x_{i, t}$ и пространственным переменным $x_{i, p}(2)$ - (3) входят во все уравнения, которые определяют кинематические, энергетические или иные характеристики движения. Компоненты векторов скорости $v_{i}$ и ускорения $w_{i}$ частиц определяют субстанциональные производные от уравнений (1) по времени:

$$
v_{i}=d x_{i} / d t \equiv x_{i, t} \quad w_{i}=d v_{i} / d t=d x_{t}^{2} / d t^{2} \equiv x_{i, t t} .
$$

Для сокращения записей производных использованы нижние индексы при обозначении функций, как в уравнениях (11) и ниже, которые соответствуют дифференцированию по указанным в индексах переменным. Если функция, например, компоненты скорости $v_{i}$ или напряжения $\sigma_{\mathrm{ij}}$, уже имеют нижний индекс, переменная, по которой следует проводить дифференцирование, указана после запятой, например $v_{i, x}$.

К неудобствам использования пространства переменных Лагранжа некоторые исследователи относят необходимость применения двух операторов бесконечно малых для времени $(d)$ и пространства $(\delta)$. Однако, это мнение ошибочно. Напротив, два отличающихся по виду и смыслу оператора существенно облегчают понимание всего комплекса уравнений, относящихся к описанию любых характеристик процессов или состояний, характеризующих движение [8]. 
При анализе процессов движения в пространстве Эйлера, как правило, рассматривают поля перемещений, скоростей частиц и других характеристик движения. Для этого траектории частиц не требуются, и это позволяет не использовать переменные Лагранжа, хотя уравнения (1) всегда можно преобразовать к форме Эйлера $\alpha_{p}=\alpha_{p}\left(x_{i}, t\right)$.

В дальнейшем в качестве основных приняты поля скоростей с проекциями на оси координат $u, v, w$ :

$$
\vec{v}=u \vec{i}+v \vec{j}+w \vec{k}
$$

которые использованы для определения деформированного, напряженного состояний и энергетических характеристик процессов.

К преимуществам такого описания следует отнести хорошо развитую на протяжении нескольких столетий теорию поля [10], которая позволяет выявить проявление некоторых особенностей, например, вихри. Однако, принимая во внимание, что для оценки условий пластичности необходимо следить за изменением во времени энергетических и иных характеристик не для точек пространства наблюдателя, а для частиц [3, 7], определение производных по времени существенно усложняются. Например, для ускорений, принимая во внимание обозначения в уравнении (12), получаем (нижний индекс $t$ соответствует субстанциональным производным):

$$
\vec{v}_{t}=u_{t} \vec{i}+v_{t} \vec{j}+w_{t} \vec{k}
$$

каждую компоненту $u_{t}, v_{t}, w_{t}$ определяют 4 слагаемых, включая локальную производную по времени $f_{\tau}$, например,

$$
u_{t}=u_{\tau}+u_{x} u+u_{y} v+u_{z} w .
$$

К сожалению, в этом случае использовать компактные записи типа $x_{i, p}$ не всегда возможно. Тем не менее, как и при описании движения в форме Лагранжа, нижние индексы для различных функций будут соответствовать их дифференцированию по указанным в индексах переменным. Индекс «0» относится к исходному состоянию. Частные и полные (субстанциональные) производные по времени отличаются соответственно индексами $\tau$ и $t$. Оператор $d$ характеризует изменение любых функций, отнесенных к рассматриваемой фиксированной массе, за бесконечно малый промежуток времени $d t$. Оператор $\delta$ в рассматриваемом варианте классической механики не используется.

Рассмотрим, например, произвольную функцию $f=f(x, y, z, t)$, характеризующую некоторое свойство фиксированной частицы, например, компоненту скорости, плотность, объем или ее энергию. В соответствии с общим правилом дифференцирования неявно заданных функций субстанциональную производную определяет уравнение [8-9]:

$$
f_{t}=f_{\tau}+f_{x} u+f_{y} v+f_{z} w .
$$

Более громоздкими становятся уравнения для производных от скоростей перемещения частиц или иных функций, характеризующих их свойства, например, плотности, по направлениям осей координат, которые являются аналогами элементов тензора (2) и имеют существенное значение при исследовании процессов деформации. В частности, чтобы найти производную от скорости (13) изменения некоторого свойства $f_{t}=f_{t}(x, y, z, t)$ по направлениям, необходимо опять пользоваться правилом дифференцирования неявно заданных функций. Учитывая, что порядок определения частных производных может быть изменен, $f_{\tau x}=f_{x \tau}, f_{x y}=f_{y x}$, получаем соотношения 


$$
f_{t x}=f_{\tau x}+f_{x x} u+f_{x} u_{x}+f_{y x} v+f_{y} v_{x}+f_{z x} w+f_{z} w_{x}=f_{x t}+f_{x} u_{x}+f_{y} v_{x}+f_{z} w_{x} .
$$

Окончательные уравнения, которые будут использованы в дальнейшем, для трех координатных осей имеют вид:

$$
\begin{gathered}
f_{t x}=f_{x t}+f_{x} u_{x}+f_{y} v_{x}+f_{z} w_{x}, \\
f_{t y}=f_{y t}+f_{x} u_{y}+f_{y} v_{y}+f_{z} w_{y}, \\
f_{t z}=f_{z t}+f_{x} u_{z}+f_{y} v_{z}+f_{z} w_{z} .
\end{gathered}
$$

Для энергетической интерпретации наиболее популярного в настоящее время условия пластичности воспользуемся уравнением для потенциальной энергии деформации $U(x, y, z, t)$ рассматриваемой массы среды [4-5]:

$$
d \delta U / \delta V d t=\omega=\sigma_{x x} u_{x}+\sigma_{x y} v_{x}+\sigma_{x z} w_{x}+\sigma_{y x} u_{y}+\sigma_{y y} v_{y}+\sigma_{y z} w_{y}+\sigma_{z x} u_{z}+\sigma_{z y} v_{z}+\sigma_{z z} w_{z},
$$

где $\omega$ - удельная мощность деформации. Для симметричного тензора напряжений уравнение для мощности совпадает с обычно используемым в [5-6]. Вместе с (15), в теории пластичности используется понятие удельной потенциальной энергии деформации:

$$
a=\int_{t_{0}}^{t_{1}} \omega d t
$$

Если подынтегральная функция задана в пространстве переменных Эйлера $\omega(x, y, z, t)$, получение результата для фиксированной частицы на интервале времени $t_{0}<t<t_{1}$ связано со значительными математическими трудностями [9-10]. Пренебрегая требованием фиксации частицы, результат будет характеризовать изменение во времени удельной энергии в фиксированной точке пространства, в которой в каждый момент времени могут находиться различные частицы. Этот результат не имеет физического смысла, как например, интегральная по времени скорость всех проехавших через центр перекрестка автомобилей.

Чтобы облегчить анализ и акцентировать внимание на фиксированных частицах, а не точках пространства наблюдателя, введем новый параметр [14]:

$$
\theta=\delta U / \delta V
$$

который будем называть объемной плотностью потенциальной энергии деформации, отнесенной к объему частицы в ее текущем состоянии. При таком определении для изменения потенциальной (упругой) энергии частицы на интервале времени $t_{0}<t<t_{1}$ интегрирования не требуется, оно равно разности произведений $\theta$ и объема в конечный и начальный моменты времени:

$$
\Delta \delta U=\delta U\left(t=t_{1}\right)-\delta U\left(t=t_{0}\right)=\theta\left(t_{1}\right) \delta V\left(t_{1}\right)-\theta_{0} \delta V_{0} .
$$

Одно из преимуществ функции (16) состоит в том, что для нее можно использовать общее дифференциальное соотношение:

$$
d \delta U(t)=d(\theta \delta V)=\left(\theta_{t}+\theta d i v \vec{v}\right) \delta V d t,
$$

где $\theta_{t}+\theta d i v \vec{v}$ определяет мощность деформации $\omega(x, y, z, t)$ из уравнения (15). 
Дифференциальное соотношение (18) справедливо для любых функций, относящихся к фиксированной частице среды. Например, изменение плотности материала $\rho$ при сохранении массы $\delta m$ определяет уравнение:

$$
d \delta m(t)=d(\rho \delta V)=\left(\rho_{t}+\rho J\right) \delta V d t=0, \quad \rho_{t}=-\rho J, \quad d \rho / d t=-\rho d i v \vec{v} .
$$

Приравнивая правые части уравнений (15) и (18), получаем уравнение для объемной плотности энергии:

$$
\begin{aligned}
& \theta_{t}=\omega-\theta J=\left(\sigma_{x x}-\theta\right) u_{x}+\sigma_{x y} v_{x}+\sigma_{x z} w_{x}+ \\
& +\sigma_{y x} u_{y}+\left(\sigma_{y y}-\theta\right) v_{y}+\sigma_{y z} w_{y}+\sigma_{z x} u_{z}+\sigma_{z y} v_{z}+\left(\sigma_{z z}-\theta\right) w_{z} .
\end{aligned}
$$

Если компоненты тензоров напряжений $\sigma_{\mathrm{ij}}$ и скорости деформации $u_{i}$ или зависимость между ними известны, уравнение может быть проинтегрировано.

Обращая внимание на наличие одинаковых множителей в уравнениях (14) и (19), будем искать функцию $\theta$ в общем случае деформации в виде:

$$
\theta=\Phi-\varphi \operatorname{div} \vec{\psi},
$$

где по существу предполагается, что из скалярной функции $\theta$ может быть выделена составляющая, определяемая дивергенцией некоторого вектора:

$$
\vec{\psi}=\xi \vec{i}+\eta \vec{j}+\zeta \vec{k}
$$

Дифференцируя уравнение (20) по времени с использованием соотношений (14):

$$
\begin{aligned}
& \theta_{t}=\Phi_{t}-\varphi_{t} \operatorname{div} \vec{\psi}-\varphi \operatorname{div} \vec{\psi}_{t}+\varphi\left(\xi_{x} u_{x}+\xi_{y} v_{x}+\xi_{z} w_{x}+\right. \\
& \left.+\eta_{x} u_{y}+\eta_{y} v_{y}+\eta_{z} w_{y}+\zeta_{x} u_{z}+\zeta_{y} v_{z}+\zeta_{z} w_{z}\right)
\end{aligned}
$$

и сравнивая правую часть с исходным уравнением (19), находим, что решение в форме (20) существует, если функции $\Phi, \varphi$ и (21) связаны дифференциальным соотношением:

$$
\Phi_{t}=\varphi_{t} \operatorname{div} \vec{\psi}+\varphi \operatorname{div} \vec{\psi}_{t}
$$

при условии:

$$
\frac{\sigma_{x x}-\theta}{\xi_{x}}=\frac{\sigma_{x y}}{\xi_{y}}=\frac{\sigma_{x z}}{\xi_{z}}=\frac{\sigma_{y x}}{\eta_{x}}=\frac{\sigma_{y y}-\theta}{\eta_{y}}=\frac{\sigma_{y z}}{\eta_{z}}=\frac{\sigma_{z x}}{\zeta_{x}}=\frac{\sigma_{z y}}{\zeta_{y}}=\frac{\sigma_{z z}-\theta}{\zeta_{z}}=\varphi,
$$

откуда следует:

$$
\varphi \operatorname{div} \vec{\psi}=\sigma_{x x}+\sigma_{y y}+\sigma_{z z}-3 \theta .
$$

С учетом (22)-(25)функцию (15) можно выразить через напряжения $\sigma_{x x}, \sigma_{y y}, \sigma_{z z}$ и функцию Ф:

$$
\theta=0,5\left(\sigma_{x x}+\sigma_{y y}+\sigma_{z z}-\Phi\right)=0,5(3 \sigma-\Phi) .
$$

Таким образом, объемная плотность энергии зависит от среднего напряжения Коши $\sigma$, а функции (21), Ф и ф позволяют учитывать анизотропию и неоднородность материала. В частности, функция Ф определяет выбор начала отсчета шкалы средних напряжений, как 
это предусмотрено в энергетической модели механики [15]. Вектора (21) и (12)в общем случае могут отличаться скалярным множителем $\chi$

$$
\vec{\psi}=\chi \vec{v} .
$$

В простейшем случае $\chi$ можно принять константой, в том числе $\chi=1$. При $\chi=$ const coотношения (24) близки к используемым в теории пластического течения изотропных материалов [5-6], а функция $\varphi$ с точностью до множителя $\chi$ совпадает с диссипативной функцией $\lambda$

$$
\frac{\sigma_{x x}-\theta}{x_{t x}}=\frac{\sigma_{x y}}{x_{t y}}=\frac{\sigma_{x z}}{x_{t z}}=\frac{\sigma_{y x}}{y_{t x}}=\frac{\sigma_{y y}-\theta}{y_{t y}}=\frac{\sigma_{y z}}{y_{t z}}=\frac{\sigma_{z x}}{z_{t x}}=\frac{\sigma_{z y}}{z_{t y}}=\frac{\sigma_{z z}-\theta}{z_{t z}}=\chi \varphi=\lambda .
$$

Для симметричного тензора напряжений и несжимаемой среды с учетом (27) $\operatorname{div} \vec{\psi}=\chi d i v \vec{v}=0$ и вместо (26) получаем

$$
\theta=\Phi=(1 / 3)\left(\sigma_{x x}+\sigma_{y y}+\sigma_{z z}\right)=\sigma,
$$

соотношения (28) переходят в известные уравнения Леви-Мизеса [2, 5].

Если $\lambda_{t i}-\lambda_{i t}=\lambda_{i} J$, дифференциальные уравнения движения [15] преобразуются к виду:

$$
\operatorname{grad} \Phi=\rho \vec{v}_{t}
$$

и совпадают с известными уравнениями движения Ньютона. Для несжимаемой среды с учетом $\Phi=$ б вместо (30) получаем:

$$
\operatorname{grad} \sigma=\rho \vec{v}_{t}
$$

Из уравнения (26) следует, что функция Ф может зависеть от различных факторов, например, температуры, которые изменяют объемную плотность энергии без изменения суммы напряжений $\left(\sigma_{x x}+\sigma_{y y}+\sigma_{z z}\right)$. С другой стороны, эта функция зависит от выбора системы отсчета для напряжений. Принято считать, что в исходном состоянии напряжения отсутствуют, хотя они, как правило, действуют в материалах и определяются силами межатомного взаимодействия, поверхностным натяжением и другими факторами. Для обычной шкалы средних напряжений, используемой в классической механике деформируемого твердого тела, $\Phi=0$, однако в энергетической модели механики [8-9] рекомендовано использовать новую шкалу, в которой $\Phi=3 K$, где $K$ - модуль объемной упругости. Функция $\theta$ может учитывать эти факторы, так как они определяют внутреннюю энергию частицы (среды). Если в материале в исходном состоянии действуют некоторые напряжения $\left(\sigma_{x x}\right)_{0},\left(\sigma_{y y}\right)_{0},\left(\sigma_{z z}\right)_{0}$, исходное значение функции $\theta$ составляет:

$$
\theta_{0}=0,5\left[\left(\sigma_{x x}\right)_{0}+\left(\sigma_{y y}\right)_{0}+\left(\sigma_{z z}\right)_{0}-\Phi\right],
$$

тогда при отсчете напряжений от этого исходного состояния функция Ф может быть скорректирована к виду:

$$
\Phi=\Phi_{0}-\left[\left(\sigma_{x x}\right)_{0}+\left(\sigma_{y y}\right)_{0}+\left(\sigma_{z z}\right)_{0}\right]
$$

При таком выборе не только приращения, но и величина потенциальной (упругой) энергии, накопленной в системе, не зависит от способа вычисления и изменения схемы нагружения в процессе деформации. Принимая во внимание (19), для мощности деформации получаем: 


$$
\omega=\theta_{t}+\theta J=\omega_{o}+\omega_{\phi} .
$$

В теории пластичности удельную мощность и энергию деформации обычно раскладывают на составляющие, считая, что произведение шаровых тензоров напряжений, деформаций или скоростей деформаций соответствуют затратам на изменение объема, а произведение девиаторов - на изменение формы $[1,2]$. С точки зрения приведенных выше уравнений, такая интерпретация не является точной, так как при записи объемной плотности энергии (17) через среднее напряжение $\sigma=(1 / 3)\left(\sigma_{x x}+\sigma_{y y}+\sigma_{z z}\right)$ для удельных мощностей на изменение объема $\omega_{о}$ и формы $\omega_{\phi}$ из уравнения (18) получаем:

$$
\omega_{o}=\theta J-(1 / 3)(\theta-\Phi) J, \quad \omega_{\phi}=\theta_{t}+(1 / 3)(\theta-\Phi) J,
$$

отличие состоит в различном характере учета абсолютного значения и скорости изменения

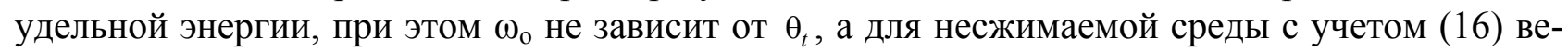
личина $\omega_{\phi}$ совпадает со скоростью изменения $\theta_{t}$.

Известный интеграл уравнений (19) позволяет описать поведение среды при различных условиях нагружения. Значение функции Ф можно определить на основе анализа процессов, хорошо изученных экспериментально. Например, при гидростатическом нагружении, когда $\sigma_{x x}=\sigma_{y y}=\sigma_{z z}=\sigma$, из уравнения (18) находим:

$$
d \theta \delta V=\sigma J \delta V d t-\theta d \delta V=(\sigma-\theta) d \delta V .
$$

С учетом объемной плотности энергии при рассматриваемых условиях $\theta=0,5(3 \sigma-\Phi)$ уравнение (31) преобразуется к виду:

$$
\frac{d(3 \sigma-\Phi)}{\sigma-\Phi}+\frac{d \delta V}{\delta V}=0
$$

Принимая $\Phi=$ const и начальные условия $\sigma=0$ при $\delta V=\delta V_{0}$, после интегрирования находим:

$$
\theta=\Phi\left(1-\frac{3}{2} R^{-1}\right)=\Phi\left(1-\frac{3}{2} \sqrt[3]{\frac{\rho}{\rho_{0}}}\right), \quad \frac{\delta V_{0}}{\delta V}=\left(1-\frac{\sigma}{\Phi}\right)^{3}
$$

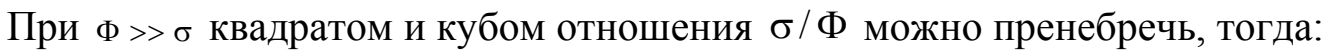

$$
\sigma=\Phi \frac{\delta V-\delta V_{0}}{3 \delta V}
$$

Сравнение результата с известным законом упругого изменения объема

$$
\sigma=K \frac{\delta V-\delta V_{0}}{\delta V_{0}}
$$

показывает, что уравнения (33) и (34) совпадают, если считать, что в исходном состоянии в металлах действуют, как отмечено выше, сжимающие напряжения (при $\Phi_{0}=0$ ), равные модулю объемной упругости:

$$
\Phi=3 K
$$


Уравнение (33) является приближенным по отношению к уравнению (32), особенно в области высоких давлений. Например, для железа при К = 169ГПа изменение объема более точно описывает уравнение:

$$
R=1-5,286^{*} 10^{-6} \sigma+1,17 * 10^{-10} \sigma^{2}-0,8 * 10^{-17} \sigma^{3} .
$$

Для этого же материала на основе более четырехсот измерений в диапазоне давлений до 300 ГПа найдена зависимость [16]:

$$
R=1+5,286^{*} 10^{-6} \sigma+0,8 * 10^{-10} \sigma^{2}
$$

которая отличается от приведенной (32) с учетом (35) на всем диапазоне давлений более чем на $0,016 \%$.

Можно предполагать, что условие (35) достаточно точно описывает поведение металлов в различных условиях нагружения. В частности, при линейном растяжении работа внешних сил производится за счет напряжений только в одном направлении, например, $\sigma_{x x} \neq 0$, при этом объемная плотность энергии и мощность в любой момент составляют:

$$
\theta=0,5\left(\sigma_{x x}-\Phi\right), \quad \omega=\sigma_{x x} u_{x} .
$$

Из уравнений (15) и (18) находим:

$$
d\left[\left(\sigma_{x x}-\Phi\right) \delta V\right]=2 \sigma_{x x} u_{x} \delta V d t,
$$

или, с учетом $d \delta V=J \delta V d t$,

$$
\left[\sigma_{x x}\left(J-2 u_{x}\right)-\Phi J\right] d t+d\left(\sigma_{x x}-\Phi\right)=0
$$

Принимая среду изотропной, а коэффициент Пуассона постоянным

$$
\frac{v_{y}}{u_{x}}=\frac{w_{z}}{u_{x}}=\frac{\varepsilon_{y}}{\varepsilon_{x}}=\frac{\varepsilon_{z}}{\varepsilon_{x}}=-\mu,
$$

и учитывая, что при линейном растяжении за счет $x_{\beta}=x_{\gamma}=0$ произведение $u_{x} d t$ определяет приращение деформации по мере Генки [5-7]:

$$
u_{x} d t=\frac{1}{R} x_{t \alpha} \tilde{x}_{\alpha} d t=\frac{1}{R} \tilde{x}_{\alpha} d x_{\alpha}=\frac{1}{x_{\alpha}} d x_{\alpha}=d \ln x_{\alpha}=d \varepsilon_{x},
$$

уравнение (36) записываем в виде:

$$
\left[\sigma_{x x}(1+2 \mu)+\Phi(1-2 \mu)\right] d \varepsilon_{x}=d\left(\sigma_{x x}-\Phi\right) .
$$

При $\Phi=$ const и начальном условии $\sigma_{x x}=0$ при $\varepsilon_{x}=0$ получаем:

$$
\sigma_{x x}=\Phi \frac{1-2 \mu}{1+2 \mu}\left[e^{\varepsilon_{x}(1+2 \mu)}-1\right]
$$

Если деформации малы, тогда $\exp \left[\varepsilon_{x}(1+2 \mu)\right] \approx 1+\varepsilon_{x}(1+2 \mu)$, с учетом соотношения (35) и известной зависимости между модулями упругости $3 K(1-2 \mu)=E$ уравнение (37) совпадает с законом Гука для линейного растяжения: 


$$
\sigma_{x x}=E \varepsilon_{x} .
$$

Выявленная связь объемной плотности энергии $\theta$ с первым инвариантом тензора напряжений даже в простейшем случае (29) не противоречит известному уравнению для удельной энергии упругой деформации [8-9]^

$$
\theta=\frac{1}{2 R}\left(\tau_{\alpha x} x_{\alpha}+\tau_{\beta x} x_{\beta}+\tau_{\gamma x} x_{\gamma}+\tau_{\alpha y} y_{\alpha}+\tau_{\beta y} y_{\beta}+\tau_{\gamma y} y_{\gamma}+\tau_{\alpha z} z_{\alpha}+\tau_{\beta z} z_{\beta}+\tau_{\gamma z} z_{\gamma}\right)-\frac{1}{2} \Phi
$$

Переходя к тензору линейных деформаций $[10,17]$ :

$$
\varepsilon_{x}=x_{\alpha}-1, \quad \varepsilon_{y}=y_{\beta}-1, \quad \varepsilon_{z}=z_{\gamma}-1, \quad \gamma_{x y}=x_{\beta}+y_{\alpha}, \quad \gamma_{y z}=y_{\gamma}+z_{\beta}, \quad \gamma_{z x}=z_{\alpha}+x_{\gamma},
$$

для упругой области вместо выражения (38) при $\tau_{\alpha y}=\tau_{\alpha z}=\tau_{\beta x}=\tau_{\beta z}=\ldots$ получаем:

$$
\theta=\frac{1}{2 R}\left(\tau_{\alpha x} \varepsilon_{x}+\tau_{\beta y} \varepsilon_{y}+\tau_{\gamma z} \varepsilon_{z}+\tau_{x y} \gamma_{x y}+\tau_{y z} \gamma_{y z}+\tau_{z x} \gamma_{z x}\right)+\frac{1}{2 R}\left(\tau_{\alpha x}+\tau_{\beta y}+\tau_{\gamma z}-\Phi R\right)
$$

Приращение потенциальной (упругой) энергии в процессе деформации определяет уравнение (17), которое с учетом (40) преобразуется к виду:

$$
\begin{aligned}
& \delta U(t)-\delta U_{0}=0,5\left(\tau_{\alpha x} \varepsilon_{x}+\tau_{\beta y} \varepsilon_{y}+\tau_{\gamma z} \varepsilon_{z}+\tau_{x y} \gamma_{x y}+\tau_{y z} \gamma_{y z}+\tau_{z x} \gamma_{z x}\right) \delta V_{0}+ \\
& +0,5\left(\tau_{\alpha x}+\tau_{\beta y}+\tau_{\gamma z}-\Phi R\right) \delta V_{0}+0,5 \Phi \delta V_{0} .
\end{aligned}
$$

Так как $\tau_{\alpha x}+\tau_{\beta y}+\tau_{\gamma z}=3 \sigma$, сумма двух последних слагаемых при условии (35), в соответствии с законом упругого изменения объема (34), обращается в 0, а массовая плотность энергии отличается от билинейной формы представления удельной энергии упругой деформации лишь на постоянную величину, определяемую последним слагаемым.

Полученное уравнение для объемной плотности энергии согласуется с известными уравнениями состояния газовых и других сред, а расчет приращения потенциальной энергии в области упругих деформаций дает результаты, не отличающиеся от общепринятых. Вместе с тем, использование удельной потенциальной энергии деформации $a=\int \omega d t$, если при интегрировании не выполнены условия фиксирования материальной частицы, не характеризует энергетическое состояние среды и не может использоваться в критериях пластичности и характеристиках видов напряженного состояния. Широко используемая в теориях упругости и пластичности интенсивность касательных напряжений [3-7]:

$$
\tau_{e}^{2}=\frac{1}{6}\left[\left(\sigma_{x x}-\sigma_{y y}\right)^{2}+\left(\sigma_{y y}-\sigma_{z z}\right)^{2}+\left(\sigma_{z z}-\sigma_{x x}\right)^{2}\right]+\tau_{x y}^{2}+\tau_{y z}^{2}+\tau_{z x}^{2},
$$

как и мощность $\omega_{\phi}$ :

$$
\omega_{\phi}=\tau_{e} s_{e}=\frac{1}{2} \lambda s_{e}^{2}=\frac{1}{2} \lambda\left[2 \operatorname{div} \vec{v}_{t}+(\operatorname{rot} \vec{v})^{2}-2 J_{t}-2 / 3\left(R_{t} / R\right)^{2}\right]=\frac{2}{\lambda} \tau_{e}^{2},
$$

зависят от скорости изменения объемной плотности энергии, в частности:

$$
\tau_{e}^{2}=0,5 \lambda \theta_{t}-(1 / 6)(\Phi-\theta)^{2}+(1 / 4) \lambda^{2}(r o t \vec{v})^{2} .
$$


Принимая, что интенсивность касательных напряжений $\tau_{\mathrm{e}}$ определяет в основном скорость изменения $\theta_{t}$, а среднее напряжение $\sigma$ - накопленную (упругую) энергию $\theta$, используемые в настоящее время критерии вида напряженного состояния $\tau_{e} / \sigma$ [3-4] и условие пластичности $a_{\phi}=$ const [5-6] можно интерпретировать как существование предельной скорости изменения или передачи энергии для каждого энергетического состояния. Если фактическое значение $\tau_{\mathrm{e}}$ превысит предельное значение, избыточная энергия реализуется в виде повышения температуры как при тепловом ударе.

Можно предполагать, что скалярная функция $\chi$, как и упоминаемые выше $\Phi, \varphi$, позволяет учитывать анизотропию и неоднородность материала. Для любой скалярной функции $\chi$ с учетом уравнений (34), (35), (39) скорость изменения объемной плотности энергии может быть представлена в виде:

$$
\theta_{t}=\varphi\left[0,5 \chi S^{2}+\operatorname{grad}(\chi) \cdot \vec{v}_{t}\right]
$$

Для однородных изотропных сред при $\chi=$ const получаем:

$$
\theta_{t}=0,5 \varphi \chi S^{2}=\varphi \chi\left(u_{x}^{2}+v_{y}^{2}+w_{z}^{2}+2 u_{y} v_{x}+2 v_{z} w_{y}+2 w_{x} u_{z}\right) \text {, }
$$

где величина $S^{2}$ характеризует дивергенцию вектора ускорения $\vec{v}_{t}$ и изменения плотности среды $\rho:$

$$
S^{2}=S_{e}^{2}+2 / 3(\operatorname{div} \vec{v})^{2}-(\operatorname{rot} \vec{v})^{2}=2\left[\operatorname{div} \vec{v}_{t}+\left(\rho_{t} / \rho\right)_{t}\right]
$$

Для несжимаемой среды получаем удвоенную дивергенцию вектора ускорения, а если к тому же поле скоростей потенциально $\operatorname{rot} \vec{v}=0$, тогда $S$ совпадает с обычно используемой интенсивностью скорости деформации сдвига $S_{\mathrm{e}}$ :

$$
S_{e}^{2}=\frac{2}{3}\left[\left(u_{x}-v_{y}\right)^{2}+\left(v_{y}-w_{z}\right)^{2}+\left(w_{z}-u_{x}\right)^{2}\right]+\left(u_{y}+v_{x}\right)^{2}+\left(v_{z}+w_{y}\right)^{2}+\left(w_{x}+u_{z}\right)^{2}
$$

и характеризует мощность деформации:

$$
\omega=0,5 \lambda S^{2} .
$$

Уравнения для ускорения относительного изменения объема позволяют выразить интенсивность скорости деформации сдвига (42), интегрирование которой по времени дает возможность определить параметр упрочнения Одквиста [6], через инвариантные характеристики поля скоростей и ускорений:

$$
S_{e}^{2}=2 \operatorname{div} \vec{v}_{t}+(r o t \vec{v})^{2}-2 J_{t}-2 / 3 J^{2}
$$

Таким образом, в процессах деформации, когда $S_{e}^{2} \geq 0$, энергетические затраты и упрочнение материала определяются вихревым характером поля скоростей, сжимаемостью среды и дивергенцией вектора ускорения, которые, как правило, не учитываются при решении задач обработки металлов давлением. Из уравнения (18) следует, что при одних и тех же энергетических затратах ламинарное поле скоростей может переходить в вихревое и наоборот. 


\section{ВЫВОДЫ}

Переход от обратимых деформаций к необратимым обязательно сопровождается диссипацией: энергия деформации частиц преобразуется в тепло, которое выделяется в окружающее пространство и может быть зафиксировано в виде повышения температуры.

Описать механизм перехода от обратимых деформаций к необратимым позволяет уравнение, отражающее процесс преобразования упомянутых видов энергии при безусловном выполнении закона сохранения. Основой для этого является уравнение (4), все слагаемые которого должны иметь одинаковую размерность [Па]. Инварианты $\xi_{5}, \xi_{6} \ldots \xi_{13}$, относящиеся к области деформаций, имеют различную размерность, в соответствии с которой размерность коэффициентов $k_{5}, k_{6}, \ldots k_{13}$ также должна отличаться. Учитывая, что физические или механические свойства с размерностью [Па $\left.c^{2}\right]$ и $\left[\right.$ Па $\left.c^{3}\right]$ в современной механике деформируемого твердого тела не используются, целесообразно инварианты $\xi_{9}, \xi_{10}$ заменить на подынтегральные функции инвариантов $\xi_{12}, \xi_{13}$, которые имеют размерность [1/c]. Отличие этих инвариантов от других состоит в том, что они характеризуют не состояния, а процесс. Если движение прекращается, эти инварианты равны 0.

Инвариант $\xi_{8}$ не изменяется при повороте осей координат, но зависит от поворота частицы как жесткого целого, это не должно отражаться на энергии деформации и поэтому $\xi_{8}$ из уравнения (10) должен быть исключен. Два инварианта $\xi_{9}, \xi_{10}$ позволяют описать механизм преобразования упомянутых выше видов энергии в виде трех слагаемых:

$$
k_{9} S_{e}+k_{10} R_{t}+c \rho_{0} \Delta T=0
$$

в котором в качестве инварианта $\xi_{9}$ использована интенсивность скорости деформации сдвига (42). Принимая в качестве коэффициента $k_{9}$ диссипативную функцию (28) $k_{9}=\lambda$, с учетом $\lambda S_{e}=\tau_{e}$, энергетическое условие перехода энергии деформации в температуру принимает вид:

$$
\tau_{e}+k_{10} R_{t}+c \rho_{0} \Delta T=0
$$

Не анализируя возможные варианты представления свойства $k_{10}$, на основе полученного уравнения можно утверждать, что энергия $\delta E_{10}=k_{10} R_{t} \delta V_{0}$, выделяемая частицей при изменении ее объема, может расходоваться либо на увеличение интенсивности напряжений до значения $\tau_{e}=\left|k_{10} R_{t}\right|$ без изменения температуры частицы $(\Delta T=0)$, если $\tau_{e}<\tau_{s}$, либо на повышение температуры ( $\Delta T>0)$, если интенсивность напряжений достигла предельного значения $\tau_{e}=\tau_{s}$.

Приведенные соотношения не противоречат известным уравнениям механики деформируемого твердого тела, дополняют их, устанавливают связь уравнений механики и термодинамики. Они учитывают сжимаемость среды, вихревой характер поля скоростей и ускорений, а, следовательно, могут способствовать дальнейшему развитию теории пластичности и обработки металлов давлением.

\section{СПИСОК ИСПОЛЬЗОВАННОЙ ЛИТЕРАТУРЫ}

1. Зибель Э. Обработка металлов в пластическом состоянии. Москва. Металлургиздат. 1934. 201 с.

2. Сторожев М.В., Попов Е.А. Теория обработки металлов давлением: учебник для вузов. Москва: Машиностроение. 1977. 287 с.

3. Колмогоров В.Л. Механика обработки металлов давлением: учебник для вузов. Москва: Металлургия. 1986. 687 с.

4. Соколов Л.Н., Алієв І.С, Марков О.Є., Алієва Л.І. Технологія кування: підручник для ВНЗ. Краматорськ: ДДМА. 2011. 268 с.

5. Томленов А.Д. Теория пластического деформирования металлов. Москва: Металлургия. 1972. 408 с. 
6. Качанов Л.М. Основы теории пластичности. Москва: Наука. 1969. 420 с.

7. Прагер В. Введение в механику сплошных сред. Москва: Иностранная литература. 1963. 312 с.

8. Алюшин Ю.А. Новая концепция в механике на основе понятий пространство, время и энергия. Физическая мезомеханика. 2018. Т. 21. 3. С. 59-69.

9. Алюшин Ю.А. Энергетические основы механики. Lambert Academic Publishing. 2016. 281 с.

10. Работнов Ю.Н. Механика деформируемого твердого тела. Москва: Физматгиз. 1979. 744 с.

11. Фейнман Р., Лейтон Р., Сэндс М. Фейнмановские лекции по физике. Москва: Мир. 1965. 219 с.

12. Алюшин Ю.А. Энергетические особенности свободных колебаний в упругих телах. Физическая мезомеханика. 2019. Т. 22. 3. С. 77-87.

13. Алюшин Ю.А. Энергетическая основа резонанса в упругих телах. Физическая мезомеханика. 2019. T. 22. 5. C. $42-53$.

14. Алюшин Ю.А. Исследование процессов обработки металлов давлением с помощью кинематически возможных полей скоростей: учеб. пособие. Ростов: РИСХМ. 1978. 98 с.

15. Алюшин Ю.А. Энергетические основы механики: учеб. пособие для вузов. Москва: Машиностроение. 1999. 192 с.

16. Кэй Дж., Лэби Т. Таблицы физических и химических постоянных. Москва: ГИФМЛ. 1962. 246 с.

17. Лурье А.И. Теория упругости. Москва: Наука. 1970. 148 с.

\section{REFERENCES}

1. Siebel E. Processing of metals in a plastic state. Moscow: Metallurgizdat. 1934, 201 p. (in Russian).

2. Storozhev M.V., Popov E.A. Theory of metal forming. textbook for high schools. Moscow: Mechanical Engineering. 1977, 287 p. (in Russian).

3. Kolmogorov V.L. Mechanics of metal forming: a textbook for high schools. Moscow: Metallurgy. 1986, 687 p. (in Russian).

4. Sokolov L.N., Aliiev I.S., Markov O.E., Aliieva L.I. Forging technology: a textbook for high schools. Kramatorsk: DSEA. 2011, 268 p. (in Ukrainian).

5. Tomlenov A.D. Theory of plastic deformation of metals. Moscow: Metallurgy. 1972, 408 p. (in Russian).

6. Kachanov L.M. Fundamentals of the theory of plasticity. Moscow: Nauka, 1969. 420 p. (in Russian). (in Russian).

7. Prager V. Introduction to the mechanics of continuous media. Moscow: Foreign literature. 1963,312 p.

8. Alyushin Yu.A. A new concept in mechanics based on the concepts of space, time and energy. Physical mesomechanics journal. 2018, 21, 3, pp. 59-69 (in Russian).

9. Alyushin Yu.A. Energy fundamentals of mechanics. Lambert Academic Publishing. 2016, 281 p. (in Russian).

10. Rabotnov Yu.N. Mechanics of a deformable solid. Moscow: Fizmatgiz. 1979, 744 p. (in Russian).

11. Feynman R., Leighton R., Sands M. Feynman Lectures in Physics. Moscow: Mir. 1965, 219 p. (in Russian).

12. Alyushin Yu.A. Energy features of free vibrations in elastic bodies. Physical mesomechanics journal. 2019, 22, 3, pp. 77-87 (in Russian).

13. Alyushin Yu.A. The energy basis of resonance in elastic bodies. Physical mesomechanics journal. 2019, 22, 5, pp. 42-53. (in Russian).

14. Alyushin Yu.A. The study of metal forming processes using kinematically possible velocity fields: textbook. Rostov on Don: RISHM. 1978, 98 p. (in Russian).

15. Alyushin Yu.A. Energy fundamentals of mechanics: textbook. Moscow: Mechanical Engineering. 1999, 192 p. (in Russian).

16. Kay J., Laby T. Tables of physical and chemical constants. Moscow: GIFML. 1962, 246 p. (in Russian).

17. Lurie A.I. Theory of elasticity. Moscow: Nauka. 1970, 148 p. (in Russian).

Алюшин Ю. А. - д-р техн. наук, проф. НИТУ МИСиС.

НИТУ МИСИС - Национальный исследовательский технологический университет МИСИС, г. Москва, РФ.

E-mail: alyushin7@gmail.com. 